\title{
Anesthesia, the aging brain, and the surgical patient
}

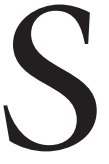

ORRY to bring you disquieting news but we're not getting any younger. According to the recent US census, there were 4.2 million Americans age 85 or older in 2000, an increase of $30 \%$ since 1990 , and those 75 to 84 numbered 12.4 million ( $v s 10$ million in 1990). The trends in Canada are no different. Many of these people will require anesthesia and surgery. Therefore, inasmuch as the brain is the main target organ for anesthetic and adjuvant drugs, age-associated changes in the brain have important anesthetic implications for this patient population.

\section{The aging brain: "normal" aging}

I. Morphologic, physiologic, and biochemical changes

Average brain weight and neuronal number inevitably decline with age beginning in young adulthood and the decline accelerates after age $60 .{ }^{1}$ As a result, the ratio of brain volume to skull volume, which is normally about $95 \%$, declines to about $80 \%$ in nonogenerians and ventricular volume triples. However, quantification of the extent of neuronal loss with age is imprecise and complicated by technical factors and substantial regional variability. Indeed, newer data indicate the magnitude of age-related neuronal loss is much more modest than the $15 \%$ to $50 \%$ estimates of earlier studies. Even so, age also affects the business end of a neuron - the dendrites and synapses. There are age-associated decreases in neuronal size, loss of complexity of the dendritic tree, and a reduced number of synapses. ${ }^{1}$ In contrast, the physiology of the cerebral circulation appears to be remarkably normal in the healthy aged. Absolute global cerebral blood flow $(\mathrm{CBF})$ is decreased about $10-20 \%$, not because of "hardening of the arteries", but because there is less brain mass to perfuse. ${ }^{2}$ Therefore, the lower CBF appears to be a consequence of reduced metabolic demand, not a cause of it. In general, although not as thoroughly investigated as one might imagine, $\mathrm{CBF}$ and $\mathrm{CMRO}_{2}$ remain tightly coupled in aging, autoregulation seems to be preserved, and responsiveness to $\mathrm{CO}_{2}$ and hypoxemia are probably reasonably normal. Finally, numerous neurotransmitter systems are altered during aging. ${ }^{3}$ For example, dopamine uptake sites, transporters, and levels are reduced, as are cortical serotonergic, $\alpha_{2}$ and $\beta_{1}$, and GABA binding sites. Markers of central cholinergic activity also decrease, which is a finding of particular significance since failure of cholinergic neurotransmission is a central feature of Alzheimer's disease (AD) ${ }^{4}$ It would be safe to say, therefore, that nearly every aspect of the brain changes with age.

But all is not bad. There is evidence, for example, that dendritic complexity and growth can increase in cognitively normal octogenarians, ${ }^{1}$ suggesting that neuronal mechanisms crucial for learning and memory are retained in the aged but healthy central nervous system (CNS). Moreover, counter to what many of us learned in medical school, the adult brain makes new neurons and this neurogenesis continues into late old age. ${ }^{5}$ What's more, these new neurons get incorporated into memory circuits. ${ }^{6}$ As one can imagine, there is much excitement about this in the neuroscience world because it provides hope that enhancing neurogenesis might be a way to attenuate or reverse age-related cognitive decline. Consistent with this, there is evidence that simple things like physical exercise and intellectual activity maintain and improve cognitive functioning in elders. ${ }^{7}$ So, as it turns out, the adage "use it or lose it" is apt even - or especially — for the brain!

\section{Cognitive changes}

What does all this mean for brain function? Intellectual decline does not invariably accompany aging but it is common. Approximately $5 \%$ of persons over age 65 suffer from dementia, which is always a consequence of serious illness, and more subtle cognitive impairment is detectable in nearly two-thirds of all "normal" older

From the Department of Anesthesiology, Harvard Medical School, Brigham \& Women's Hospital, Boston, Massachusetts, USA.

Address correspondence to: Dr. Gregory Crosby, Department of Anesthesiology, Brigham \& Women's Hospital, 75 Francis St, Boston, MA 02115, USA. Phone: 617-732-7330; Email: gcrosby@zeus.bwh.harvard.edu

This work was supported in part by NIH R01 AG 20253 to G. Crosby and by an American Geriatrics Society Jahnigen Award and a Harvard/Hartford Foundation New Investigator Award to D. Culley. 
people. ${ }^{8}$ Such changes can be classified as either diseaserelated secondary impairment (e.g., related to alcoholism or Alzheimer's) or as primary cognitive decline. However, the line between the two is often hazy.

There are some consistently observed changes in CNS function with "normal" aging. ${ }^{8}$ There is a slowing of reaction time and cognitive processing such that there is an inverse relationship between age and speed of motor performance. The more complex the task, the slower the response, presumably due to decreased sensory sensitivity (e.g., reduced auditory acuity) as well as slowed central processing of information. Deterioration in "fluid" intelligence (i.e., the ability to dynamically evaluate, accommodate, and respond to novel environmental events) also occurs. However, some tests used to assess intelligence are influenced by motor speed, so slowed reaction time confounds the assessment. On non-timed tests, vocabulary, verbal information, and comprehension are well maintained. "Crystallized" intelligence (i.e., accumulated knowledge) is also relatively stable into the seventh decade of life. Finally, short-term memory is often impaired. Between 30 and $50 \%$ of elders report memory complaints. Much of this impairment is in working memory - which requires not only retention but also manipulation of information. Thus, the ability to store recently processed information while simultaneously acquiring new data is compromised.

Having said all this, it is important to realize that the cognitive decline associated with normal aging is modest and variable. Moreover, as mentioned earlier, it is influenced in a positive way by physical activity and intellectual engagement. ${ }^{7}$ In fact, many active elderly individuals remain stable on measures of cognitive performance into their $80 \mathrm{~s}$, and even outperform younger but inactive persons on some tests. ${ }^{8}$ Consequently, there is no such thing as a "typical" older person from this perspective, making studies of this population difficult because chronological age is often not a predictor of cognitive capability.

\section{Pathological brain aging: dementia}

Dementia is a chronic, progressive decline in intellectual function. It can be caused by numerous systemic diseases and neurologic disorders (e.g., stroke, Parkinson's disease) but AD is both the most common cause and a prototype of pathological brain aging. ${ }^{9}$ The diagnosis of dementia requires evidence of decline from a higher cognitive level, multiple cognitive defects, among which memory impairment is essential, and the defects must interfere with social or occupational functioning. Criteria stipulate a duration of more than six months and, to exclude delirium, that consciousness be unaltered.
$\mathrm{AD}$ is frighteningly common. Ten to $15 \%$ of persons older than 65 yr develop AD and by age 85 about $30-50 \%$ will be afflicted. The diagnosis is one of exclusion and requires demonstration of neurofibrillary tangles, extracellular amyloid deposits, and neuritic plaques in the brain at postmortem examination. ${ }^{3,10}$ Growing evidence suggests these changes may be seminal events in the pathogenesis of AD and not simply markers of it. ${ }^{11}$ The brain changes seen in normal aging are present in an exaggerated form in AD. Thus, loss of brain mass occurs at a rate 2.5 times normal in $\mathrm{AD}^{12}$ and hypofunction of cholinergic neurotransmission is more pronounced, particularly in areas associated with memory and cognition. This cholinergic deficiency is a hallmark of the disease but may not, as once thought, explain the memory deficits because there is no such deficiency in the patient with mild AD. ${ }^{13}$ The list of other potential mechanisms of $\mathrm{AD}$ is lengthy and includes complement mediated inflammatory responses, oxidative damage to proteins and DNA, and alterations in the hormonal milieu. ${ }^{10,14,15}$ Although some of these are being investigated as potential sites for future therapeutic intervention there is as yet no "magic bullet" for AD.

Thus, medical therapy has little or no effect on progression of AD but aims to modify the symptoms. The mainstay of treatment still is anticholineasterases such as tacrine and donepezil, ${ }^{16}$ but vitamin E, steroids, and estrogen also have advocates. ${ }^{10,14}$ There is some evidence that these drugs may improve memory, attention deficits, and symptoms of AD such as apathy, agitation and hallucinations but none seem to prevent the disease or slow progression. ${ }^{14}$ In this regard, probably the most exciting newer developments in $\mathrm{AD}$ treatment and prevention revolve around immunization against $\beta$ amyloid and use of anti-inflammatory agents. ${ }^{14,15}$ Initial animal work with $B$ amyloid immunization showed a marked decrease in $\beta$ amyloid deposits in brain and a corresponding improvement in cognitive performance. Based on this, a human trial was initiated but was recently stopped prematurely because some patients developed an encephalitic reaction to the antigen. Because the approach seems promising, the search is now on for a different antigen that will prove safer and effective. ${ }^{14}$ However, antiinflammatory agents may be the best of what's available today. It turns out that in addition to anti-inflammatory effects, some non-steroidal antiinflammatory drugs (NSAIDS; ibruprofen but not naproxen or aspirin) decrease $B$ amyloid production. ${ }^{15}$ Consistent with this action, a few epidemiological and small clinical studies suggest regular use of certain NSAIDS reduces the risk of developing AD. Who 
knows, maybe all those anti-inflammatory agents we take for age-related aches and pains help our brain as well as our muscles and joints "feel" better!

\section{Aging, anesthesia, and postoperative cognitive dysfunction (POCD)}

It has been suspected for at least 48 years that some elderly patients may develop dementia following anesthesia and surgery. ${ }^{17}$ Concern that the elderly brain "takes a hit" during general anesthesia and surgery is better justified today but we're still far from sure about the etiology and significance of the problem. ${ }^{18}$ This is because we still know so little about the effects of anesthetics on the aged brain. The age-associated structural and functional changes described above imply, howev$\mathrm{er}$, that the aged CNS has reduced functional reserve and the current assumption is that this makes the elderly more vulnerable to the development of POCD.

\section{Delirium}

Delirium, defined as acute disturbance in consciousness and cognition that tends to fluctuate throughout the day ${ }^{19}$ is the most common form of perioperative CNS dysfunction. It is twice as common in the elderly, occurring in $10-15 \%$ of elderly general surgical patients and as many as $30-50 \%$ of those undergoing orthopedic or cardiac surgery. ${ }^{20,21}$ The mechanism is unknown but one hypothesis is that it may be related to further decreases in already low levels of neurotransmitters such as acetylcholine. Other etiologic or predisposing factors include hypoxia, drug interactions (particularly anticholinergics, benzodiazepines, and tricyclic antidepressants), alcohol abuse, pre-existing depression or dementia, and metabolic disturbances. Delirium is more than an annoyance; it is associated with longer hospital stay, higher mortality, and may even be a marker for subsequent development of dementia. ${ }^{22}$

Several anesthetic agents, including ketamine, benzodiazepines, and even propofol, can produce delirium but in the elderly the strongest association is with the anticholinergic agents atropine and scopolamine. ${ }^{19}$ This is presumably because of the baseline cholinergic deficiency and suggests that with the exception of glycopyrrolate, which does not cross the blood brain barrier, medications with anticholingeric properties should be used sparingly in these patients. Curiously, however, the type of anesthesia does not influence the risk of developing delirium. Specifically, the incidence of postoperative confusion appears to be similar regardless of whether spinal, epidural, or general anesthesia is used. ${ }^{23}$ Moreover, postoperative epidural analgesia has proven no better than the $i v$ route from this point of view. ${ }^{21}$
What can one do about perioperative delirium? First, identify patients at high risk ${ }^{24}$ and avoid or minimize some of the medications just mentioned. In addition, consider obtaining a preoperative geriatric consultation since there is some evidence that such consultation markedly reduces the incidence of delirium, particularly in high risk patients. ${ }^{25}$ Second, treat remediable causes of delirium such as hypoxemia or sepsis. Many of the factors contributing to delirium in the hospital setting are not modifiable (e.g., age of patient, type of surgery) so it is especially important to manage those that can be modified. Lastly, when prevention fails, one can manage the symptoms with agents such as haloperidol or atypical antipsychotics such as olanzapine.

\section{Prolonged POCD}

There is now substantial evidence that many elderly patients experience cognitive deterioration postoperatively. In a prospective, randomized trial of general $v s$ epidural anesthesia with sedation for total knee replacement in patients $>70 \mathrm{yr}$ of age, cognitive performance, as assessed with psychometric tests, was worse than the preoperative baseline in $4-6 \%$ of patients six months after anesthesia and surgery. ${ }^{23}$ However, this study lacked a control group for the effects of time or hospitalization alone. More convincing is a large, prospective, controlled international study $^{26}$ that demonstrated a cognitive deficit in $9.9 \%$ of patients three months postoperatively whereas only about $3 \%$ of the age-matched controls were similarly impaired. Among patients over $75 \mathrm{yr}$ of age, $14 \%$ had a persistent cognitive deficit after general anesthesia and surgery. ${ }^{26}$ Note that although postoperative cognitive impairment is not unique to the elderly, ${ }^{27}$ it does seem to be more severe and longer lasting in them. It is also important to recognize, however, that deficits were defined by performance on psychometric tests and that the social or economic significance of the impairment, if any, has not been well examined. Nevertheless, it is clear that prolonged postoperative cognitive impairment is both real and more common than previously realized. The good news is that patients seem to recover completely within one to two years or less, but this is based on data from a small subgroup of patients. ${ }^{28}$

The key question, of course, is what causes it? The short answer is that no one is sure. One old and intuitive hypothesis is that perioperative hypotension and/or hypoxia cause or exacerbate POCD. Like most simple explanations for complex problems, however, this is probably incorrect in most cases. In fact, the largest study of POCD in elderly patients ${ }^{26}$ shows that 
neither perioperative hypotension [mean arterial pressure $(\mathrm{MAP})<60 \%$ for $\geq 30 \mathrm{~min}]$ nor hypoxia $\left(\mathrm{SpO}_{2}\right.$ $\leq 80$ for more than two minutes) are predictors of cognitive decline three months postoperatively, a finding that concurs with older work. Moreover, in a direct test of the influence of controlled hypotension on cognitive outcome in elderly patients under epidural anesthesia, no early or long-term adverse effect of hypotension (MAP $45-55 \mathrm{mmHg}$ ) was observed. ${ }^{29}$ There could be some patient or procedure - specific causes or risk factors such as a genetic predisposition ${ }^{30}$ or cerebral emboli, respectively. In fact, cerebral emboli have been detected during total knee replacement using transcranial Doppler. ${ }^{31}$ Finally, there may be factors associated with surgery itself such as stress, immobility, social isolation, and/or inflammation. These are reasonable hypotheses since each factor can influence cognition and is affected by surgery and/or hospitalization but there is presently no direct evidence that they contribute to POCD.

One factor that has received little attention is general anesthesia itself. There are many reasons to speculate that general anesthesia might contribute, not the least of which is that anesthesia is obviously a profound form of CNS dysfunction. However, clinical evidence for this is sparse. First, some data indicate the risk of prolonged POCD is similar with regional and general anesthesia. Thus, in elderly patients undergoing total knee replacement, the frequency of cognitive impairment six months postoperatively was similar (4-6\%) between epidural and general anesthesia groups. ${ }^{23}$ This is consistent with previous work indicating that shorter term postoperative (one to seven days) cognitive performance in the elderly is typically no better after spinal or epidural than general anesthesia. However, iv sedation was used to supplement the regional technique ${ }^{23}$ and patients also had a surgical procedure, making it difficult to isolate the influence of anesthesia itself. To circumvent this problem, we have investigated the long-term effects of general anesthesia on learning and memory in rats and, interestingly, found that isoflurane-nitrous oxide anesthesia without surgery impairs spatial learning in aged rats for at least three to four weeks. ${ }^{32}$ Because the agents were long cleared from the brain by the time behavioural testing was begun, these data imply that anesthesia itself alters brain regions and neurochemical cascades mediating memory and that this pharmacologic footprint lasts considerably longer than previously recognized. Accordingly, the data suggest that general anesthesia could be a factor in POCD.

In conclusion, the elderly brain is very different from that of young or middle-aged persons both in terms of anatomy and neurochemistry and function. The cognitive consequences of these age-related changes are highly variable and can be influenced by general health as well as mental and physical activity. Delirium and dementia are the result of disease processes that can be unmasked or exacerbated by hospitalization and surgery. However, even apparently cognitively intact elderly patients may suffer POCD. The etiology of POCD is likely to be multifactorial, possibly including a contribution from anesthesia itself, but because the mechanisms are still uncertain it is difficult to identify prevention or treatment strategies. In particular, there is presently no scientific basis for recommending (or avoiding) a specific anesthetic agent or technique in this regard. This may change as further research is conducted on this relatively newly identified and interesting entity. In the meantime, it is reassuring that the prognosis for recovery from POCD appears to be good.

\section{References}

1 Selkoe DJ. Aging brain, aging mind. Sci Am 1992; 267: 134-42.

2 Davis SM, Ackerman RH, Correia JA, et al. Cerebral blood flow and cerebrovascular $\mathrm{CO} 2$ reactivity in stroke-age normal controls. Neurology 1983; 33: 391-9.

3 Mrak RE, Griffin ST, Grabam DI. Aging-associated changes in human brain. J Neuropathol Exp Neurol 1997; 56: 1269-75.

4 Francis PT, Palmer AM, Snape M, Wilcock GK. The cholinergic hypothesis of Alzheimer's disease: a review of progress. J Neurol Neurosurg Psychiatry 1999; 66: $137-47$.

5 Eriksson PS, Perfilieva E, Bjork-Eriksson T, et al. Neurogenesis in the adult human hippocampus. Nat Med 1998; 4: 1313-7.

6 Shors TJ, Miesegaes G, Beylin A, Zhao M, Rydel T, Gould $E$. Neurogenesis in the adult is involved in the formation of trace memories. Nature 2001; 410: 372-6.

7 Kramer AF, Hahn S, Cohen NJ, et al. Ageing, fitness and neurocognitive function. Nature 1999; 400: 418-9.

8 Keefover RW. Aging and cognition. Neurol Clin 1998; 16: 635-48.

9 Richards SS, Hendrie HC. Diagnosis, management, and treatment of Alzheimer disease: a guide for the internist. Arch Intern Med 1999; 159: 789-98.

10 Growdon JH. Biomarkers of Alzheimer disease. Arch Neurol 1999; 56: 281-3.

11 Naslund J, Haroutunian V, Mobs R, et al. Correlation between elevated levels of amyloid beta-peptide in the brain and cognitive decline. JAMA 2000; 283: 1571-7. 
12 Jack CRJ, Petersen RC, Xu , et al. Rate of medial temporal lobe atrophy in typical aging and Alzheimer's disease. Neurology 1998; 51: 993-9.

13 Davis KL, Mohs RC, Marin D, et al. Cholinergic markers in elderly patients with early signs of Alzheimer disease. JAMA 1999; 281: 1401-6.

14 Hardy J, Selkoe DJ. The amyloid hypothesis of Alzheimer's disease: progress and problems on the road to therapeutics. Science 2002; 297: 353-6.

15 Wilson CJ, Finch CE, Cohen HJ. Cytokines and cognition--the case for a head-to-toe inflammatory paradigm. J Am Geriatr Soc 2002; 50: 2041-56.

16 Whitehouse PJ. The cholinergic deficit in Alzheimer's disease. J Clin Psychiatry 1998; 59(Suppl 13): 19-22.

17 Bedford PD. Adverse cerebral effects of anaesthesia on old people. Lancet $1955 ; 259-63$.

18 Dodds C, Allison J. Postoperative cognitive deficit in the elderly surgical patient. Br J Anaesth 1998; 81: 449-62.

19 Parikh SS, Chung F. Postoperative delirium in the elderly. Anesth Analg 1995; 80: 1223-32.

20 Lipowski ZJ. Delirium in the elderly patient. N Engl J Med 1989; 320: 578-82.

21 Williams-Russo P, Urquhart BL, Sharrock NE, Charlson $M E$. Post-operative delirium: predictors and prognosis in elderly orthopedic patients. J Am Geriatr Soc 1992; 40: 759-67.

22 Rockwood K, Cosway S, Carver D, Jarrett P, Stadnyk K, Fisk J. The risk of dementia and death after delirium. Age Ageing 1999; 28: 551-6.

23 Williams-Russo P, Sharrock NE, Mattis S, Szatrowski TP, Charlson ME. Cognitive effects after epidural vs general anesthesia in older adults. A randomized trial. JAMA 1995; 274: 44-50.

24 Marcantonio ER, Goldman L, Mangione CM, et al. A clinical prediction rule for delirium after elective noncardiac surgery. JAMA 1994; 271: 134-9.

25 Marcantonio ER, Flacker JM, Wright RJ, Resnick NM. Reducing delirium after hip fracture: a randomized trial. J Am Geriatr Soc 2001; 49: 516-22.

26 Moller JT, Cluitmans P, Rasmussen LS, et al. Longterm postoperative cognitive dysfunction in the elderly ISPOCDI study. ISPOCD investigators. International Study of Post-Operative Cognitive Dysfunction. Lancet 1998; 351: 857-61.

27 Johnson T, Monk T, Rasmussen LS, et al. Postoperative cognitive dysfunction in middle-aged patients. Anesthesiology 2002; 96: 1351-7.

28 Abildstrom H, Rasmussen LS, Rentowl P, et al. Cognitive dysfunction 1-2 years after non-cardiac surgery in the elderly. ISPOCD group. International Study of Post-Operative Cognitive Dysfunction. Acta Anaesthesiol Scand 2000; 44: 1246-51.
29 Williams-Russo P, Sharrock NE, Mattis S, et al. Randomized trial of hypotensive epidural anesthesia in older adults. Anesthesiology 1999; 91: 926-35.

30 Slooter AJ, Cruts M, Kalmijn S, et al. Risk estimates of dementia by apolipoprotein E genotypes from a population-based incidence study: the Rotterdam Study. Arch Neurol 1998; 55: 964-8.

31 Sulek CA, Davies LK, Enneking FK, Gearen PA, Lobato $E B$. Cerebral microembolism diagnosed by transcranial Doppler during total knee arthroplasty: correlation with transesophageal echocardiography. Anesthesiology 1999; 91: 672-6.

32 Culley DJ, Yukhananov RY, Baxter MG, Crosby G. memory effects of general anesthesia persist for weeks in young and aged rats. Anesth Analg 2003; 96: 1004-9. 